\title{
Dispersion of low frequency plasma waves upstream of the quasi-perpendicular terrestrial bow shock
}

\author{
A. P. Dimmock ${ }^{1,2}$, M. A. Balikhin ${ }^{1}$, S. N. Walker ${ }^{1}$, and S. A. Pope ${ }^{1}$ \\ ${ }^{1}$ Department of Automatic Control and Systems Engineering, University of Sheffield, Mappin Street, Sheffield, S1 3JD, UK \\ ${ }^{2}$ Department of Physical Sciences, Embry-Riddle Aeronautical University, Daytona Beach, Florida, 32114, USA
}

Correspondence to: A. P. Dimmock (dimmocka@erau.edu)

Received: 28 March 2013 - Revised: 30 June 2013 - Accepted: 3 July 2013 - Published: 9 August 2013

\begin{abstract}
Low frequency waves in the foot of a supercritical quasi-perpendicular shock front have been observed since the very early in situ observations of the terrestrial bow shock (Guha et al., 1972). The great attention that has been devoted to these type of waves since the first observations is explained by the key role attributed to them in the processes of energy redistribution in the shock front by various theoretical models. In some models, these waves play the role of the intermediator between the ions and electrons. It is assumed that they are generated by plasma instability that exist due to the counter-streaming flows of incident and reflected ions. In the second type of models, these waves result from the evolution of the shock front itself in the quasiperiodic process of steepening and overturning of the magnetic ramp. However, the range of the observed frequencies in the spacecraft frame are not enough to distinguish the origin of the observed waves. It also requires the determination of the wave vectors and the plasma frame frequencies. Multipoint measurements within the wave coherence length are needed for an ambiguous determination of the wave vectors. In the main multi-point missions such as ISEE, AMPTE, Cluster and THEMIS, the spacecraft separation is too large for such a wave vector determination and therefore only very few case studies are published (mainly for AMPTE UKS AMPTE IRM pair). Here we present the observations of upstream low frequency waves by the Cluster spacecraft which took place on 19 February 2002. The spacecraft separation during the crossing of the bow shock was small enough to determine the wave vectors and allowed the identification of the plasma wave dispersion relation for the observed waves. Presented results are compared with whistler wave dispersion and it is shown that contrary to previous studies based on the AMPTE data, the phase velocity in the shock frame
\end{abstract}

is directed downstream. The consequences of this finding for both types of models that were developed to explain the generation of these waves are discussed.

Keywords. Magnetospheric Physics (Plasma waves and instabilities; Solar wind-magnetosphere interactions) - Space Plasma Physics (Shock waves)

\section{Introduction}

The main process that occurs at the shock front is the re-distribution of the upstream kinetic energy of directed ions motion (Sagdeev, 1966; Sagdeev and Galeev, 1969; Papadopoulos, 1985) into thermalisation and the acceleration of a fraction of the particles to very high energies. In ordinary gasodynamic shocks, the energy redistribution takes place due to the binary collisions between particles. In such a case, the front spatial scale is determined by the the length of the free path. The spatial scale of collisionless shocks is much smaller than the free path length and therefore the effect from binary collisions can be neglected. The energy redistribution takes place due to the interaction with electric and magnetic fields of the shock transition layer or due to the interaction with plasma waves generated by various plasma instabilities. The spatial scales of the shock front structure determine the type of upstream particle interactions with the shock front and a number of statistical studies of the shock front scales have been conducted (Balikhin et al., 1995; Hobara et al., 2010; Dimmock et al., 2011). However, the composition of plasma turbulence in the front is also very important because it determines the efficiency of anomalous processes of energy redistribution that occur due to interactions with plasma waves. Various plasma waves in the 
frequency range from $10^{0} \mathrm{~Hz}$ to $10^{5} \mathrm{~Hz}$ are observed in the vicinity of the quasi-perpendicular part of the terrestrial bow shock. The upper part of this range corresponds to the electromagnetic oscillations on the second harmonic of plasma frequency that are generated due to nonlinear processes that involve Langmuir waves exited at the boundary of electron foreshock due to the beam of electrons reflected from the shock front (e.g. Balikhin et al., 1989). Ion sound waves observed in the vicinity of the magnetic ramp in the frequency range from a few hundred $\mathrm{Hz}$ to a few $\mathrm{kHz}$ (e.g. Balikhin et al., 2005), that can be generated either by current driven instabilities or by the electron temperature gradient were the key element in many early theories of anomalous processes at the shock front. However, at later stages the main attention is on the lowest part of the frequency range $10^{0}-10^{1} \mathrm{~Hz}$, because in some theoretical models it was assumed that these waves can efficiently transfer energy from ions to the electrons, or can be a manifestation of the internal shock dynamics resulting in quasi-periodic steepening and overturning of the shock front itself. In some studies the low frequency turbulence observed upstream of the shock front has been considered to be the result of two stream instabilities (e.g. Tanaka and Papadopoulos, 1983; Krasnoselskikh et al., 1985; Galeev et al., 1986) where the reflection of electrons from the front of a strong quasi-perpendicular shock resulted in the generation of plasma waves. It was argued in these studies that the resulting waves can efficiently transfer energy from the ions to energise electrons. In other models like Balikhin et al. (1989), these low frequency waves were interpreted as whistler waves. These waves can reflect part of the upstream electrons and such a reflection could lead to acceleration. The statistical properties of these waves was studied extensively by Fairfield (1974) using IMP-6 data. It was suggested that these waves span time intervals of less than a few minutes. The waves can be either right- or left-handed polarised and tend to propagate at oblique angles $\left(20^{\circ}-40^{\circ}\right)$ to the upstream magnetic field. Previous studies have identified such waves as propagating in the whistler mode and their origin is typically associated with either shock macro dynamics (Krasnoselskikh, 1985) or instabilities (Wu et al., 1984; Hellinger et al., 1996).

The majority of studies related to whistler waves in close proximity to the collisionless shock front have been observed within the terrestrial environment (e.g. Fairfield, 1974; Balikhin et al., 1997). Nevertheless, these waves have been reported at many other other locations such as Venus (Orlowski and Russell, 1991) and Saturn (Orlowski et al., 1992) concluding that they are a fundamental phenomena associated with collisionless shocks. Whistler waves are believed to play a fundamental role in shock structure and therefore have attracted considerable interest in both theoretical and experimental studies. Despite this, the generation mechanism of these waves and the exact role that they play on shock structure and formation is still not completely understood. In studies such as those by Balikhin et al. (1997) whistler mode waves have been reported to be directed upstream, thus playing a role in carrying energy from the shock front. Balikhin et al. (1997) concluded that these waves were most likely generated by the macrodynamics of the shock and the property of shock nonstationarity (Tidman and Northrop, 1968). On the contrary, other mechanisms such as proton beam instabilities (Wong and Goldstein, 1988), temperature anisotropy instability and the kinetic cross stream instability (Wu et al., 1984) have been proposed to explain their presence. It has also been shown that gyro phase bunched ions which are generated by the shock have the ability to produce low and high frequency whistler waves (Gurgiolo et al., 1993). Their results imply that the low frequency waves play an important role in the gyro phase bunched ions rather than processes such as gyro phase mixing (Gurgiolo et al., 1993) which are known to operate upstream and downstream (Ofman et al., 2009) of the shock front. It is possible to correlate spacecraft observations with the output from existing theoretical models (e.g. plasma rest frame frequencies, wave vector properties and phase velocity) however, this raises another significant problem. Whistler waves in the solar wind are severely affected by the doppler shift and thus require separation of a temporal and spatial ambiguity before a true estimate of plasma rest frame frequencies can be obtained. To further complicate matters, the wavelength of such structures are typically much smaller than the spacecraft separation vectors of current multi-spacecraft missions resulting in limited cases where such studies can be performed. In most cases even identifying the correct wave mode can be an involved process. The doppler shift between frequencies in the spacecraft and plasma rest frame $(\omega)$ are related to the wave vector by a dispersion relation $\omega=\omega(\boldsymbol{K})$. This dispersion relation can be correlated with a theoretical counterpart to aid in wave mode identification, but the full wave vector cannot be measured directly from the time series measurements and therefore must be reconstructed.

Hoppe et al. (1982) applied a technique based on correlation coefficients to calculate the dispersion relation of waves upstream of the quasi-perpendicular shock front. However, the spacecraft separation in most cases was several times the wavelength of the observed waves which made identification of the wave structures in the two datasets difficult. The dispersive nature of the plasma waves also caused significant evolution of the wave structures between the measurements. As a result of this, there are significant limitations to this technique. Firstly, only one point can be plotted on the dispersion curve for each iteration and secondly, this method is more applicable to non-dispersive waves.

A more applicable technique can be used that takes advantage of the phase shift measured between waves that are observed by two closely separated spacecraft. If two satellites are separated by a vector $\boldsymbol{R}$, then the phase difference $\Delta \Psi\left(\omega_{1}\right)$ between the waves at an observed frequency $\left(\omega_{1}\right)$ can be estimated by 
$\Delta \Psi\left(\omega_{1}\right)=\boldsymbol{K}\left(\omega_{1}\right) \cdot \boldsymbol{R}=\left|\boldsymbol{K}\left(\omega_{1}\right)\right||\boldsymbol{R}| \cos \left(\Theta_{K} R\right)$

where $\Theta_{K R}$ is the angle between $\boldsymbol{R}$ and the wave vector direction $\hat{\boldsymbol{K}}$. The direction of the wave vector can be estimated from minimum variance analysis (MVA) whereas the phase shift can be determined using the $f_{1}-k_{\text {sep }}$ spectrum obtained by performing the wavelet transform. Knowledge of both $\hat{\boldsymbol{K}}$ and $\Delta \Psi\left(\omega_{1}\right)$ can be used to calculate the magnitude of the wave vector in Eq. (1). The full wave vector can then reconstructed which allows the transformation from frequencies in the spacecraft frame to the plasma rest frame. This relationship can be used to build the experimental dispersion relation which can be compared with theoretical models to provide a means of identifying the correct wave mode. The main disadvantage from this technique is that it requires the observation of well structured plasma waves between at least two relatively close spacecraft which should be less than the coherence length and ideally even smaller than the wavelength of the observed waves. If the separation is more than the coherence length of the observed waves then the dispersive property of these waves can make it difficult to identify the correct phase shift which is the foundation of this technique. Unfortunately, the conditions which permit this analysis for waves observed in the foot of a quasi-perpendicular supercritical shock are seldom with most spacecraft configurations.

To date the Cluster mission has provided over $11 \mathrm{yr}$ of high resolution plasma measurements inclusive of thousands of terrestrial bow shock crossings. In addition to this the multiprobe arrangement allows the separation of temporal and spatial variations at the shock front. Therefore, the Cluster mission presents an ideal database for uncovering the strict requirements needed to perform studies of wave dispersion upstream of the terrestrial bow shock. On 19 February 2002 the Cluster probes encounter the terrestrial bow shock with appropriate separation vectors and natural conditions that makes such a study possible.

\section{Datasets and instrumentation}

All data used in this study was recorded by the Cluster spacecraft (Escoubet et al., 1997) on 19 February 2002. The Cluster mission is comprised of 4 separate probes launched in 2001 which orbit in a temporally dependent tetrahedron configuration. Each probe is fitted with a significant array of instrumentation capable of measuring electromagnetic fields and plasma moments. The magnetic field measurements to identify the shock crossing, and which form the fundamental component for this study were provided by the spacecraft Fluxgate Magnetometer FGM instruments (Balogh et al., 1997) onboard each spacecraft. The available resolution of magnetic field data during the shock crossing and close vicinity are $22.5 \mathrm{~Hz}$ which not only allows for detailed observation of shock structure, but also permits the clear resolution of plasma waves upstream of the shock front. The upstream solar wind velocity $\left(\boldsymbol{V}_{\mathrm{u}}\right)$ was determined using measurements provided by the Cluster Ion Spectrometer (Rème et al., 1997) (CIS). The plasma density $N_{i}$ was estimated using the electron plasma frequency $\left(\omega_{\text {pe }}\right)$ provided by the WHISPER (Décréau et al., 1997) instrument, which was used to estimate the Alfvén Mach number. WHISPER is a part of wave consortium controlled by DWP instrument (Woolliscroft et al., 1997).

\section{Measurements of shock crossing on 19 February 2002: 04:02 UTC}

On 19 February 2002 the Cluster spacecraft encountered 6 crossings of the terrestrial bow shock within a time period approximately $4 \mathrm{~h}$ where during this time the separation between Cluster 3 and 4 was approximately $105 \mathrm{~km}$. The separation along the GSE $x$ direction at this time was only $32 \mathrm{~km}$ which is significantly less than usual. At 04:02:00 UTC the Cluster spacecraft traversed the bow shock at [18.9, 3.2, -1.2] $R_{E}$ where the spacecraft separation between the $\mathrm{C} 3$ and $\mathrm{C} 4$ probes was $\boldsymbol{R}_{34}=[-32.0,-41.8,-91.4] \mathrm{km}$. The position of $\mathrm{C} 3$ suggests the crossing was in close vicinity to the sub-solar point. Figure 1 shows the magnetic field profile of the shock crossing measured by the Cluster 3 spacecraft in she shock frame where E1 $=\hat{\boldsymbol{n}}, \mathrm{E} 2=\hat{\boldsymbol{n}} \times \boldsymbol{B}_{\mathrm{u}}$ and E3 $=\hat{\boldsymbol{n}} \times\left(\hat{\boldsymbol{n}} \times \boldsymbol{B}_{\mathrm{u}}\right)$. Upstream of the shock, the average magnetic field direction was $\boldsymbol{B}_{\mathrm{u}}=[-1.39,8.88,5.89] \mathrm{nT}$ $\left(\left|\boldsymbol{B}_{\mathrm{u}}\right|=10.75 \mathrm{nT}\right)$, and the solar wind velocity was measured as $\left|\boldsymbol{V}_{\mathrm{u}}\right|=356 \mathrm{~km} \mathrm{~s}^{-1}$. The WHISPER instrument measured an electron plasma frequency of $\omega_{\mathrm{pe}}=1.38 \times 10^{5} \mathrm{rad} \mathrm{s}^{-1}$. The preceding parameters resulted in an estimation of Alfvén Mach number of $M_{\mathrm{a}}=3.7$. The Farris shock model surface (Farris et al., 1991) was used to determine the shock normal direction of $\hat{\boldsymbol{n}}=[0.99,0.09,-0.03]$. The projection of the magnetic field along $\hat{\boldsymbol{n}}$ can be seen in the top panel of Fig. 1 and the small variation during the ramp in addition to the negligible offset from upstream to downstream is strong evidence of a good shock normal direction. The location of the shock crossing also suggests a shock normal direction closely aligned with the GSE $x$ direction. The angle measured between $\hat{\boldsymbol{n}}$ and $\boldsymbol{B}_{\mathrm{u}}\left(\Theta_{b n}=86.4^{\circ}\right)$ suggests that the shock has a quasi-perpendicular geometry. The magnetic field profile is also typical from past observations and simulations of the quasi-perpendicular shock front in the supercritical regime (Leroy et al., 1982). The velocity of the shock along $\hat{\boldsymbol{n}}$ was calculated as $V_{\mathrm{Sh}} \approx-32 \mathrm{~km} \mathrm{~s}^{-1}$. This was calculated by selecting the mean velocity of the spacecraft separation vectors $\boldsymbol{S}_{12}$ and $\boldsymbol{S}_{14}$ along the shock normal direction. These vectors were chosen as they were as least perpendicular to $\hat{\boldsymbol{n}}$ as possible which produced angles (velocities) of $103^{\circ}$ $\left(-31 \mathrm{~km} \mathrm{~s}^{-1}\right)$ and $112^{\circ}\left(-33 \mathrm{~km} \mathrm{~s}^{-1}\right)$, respectively. Spacecraft separation vectors which are close to perpendicular with the shock normal are not ideal as they cannot be used to 


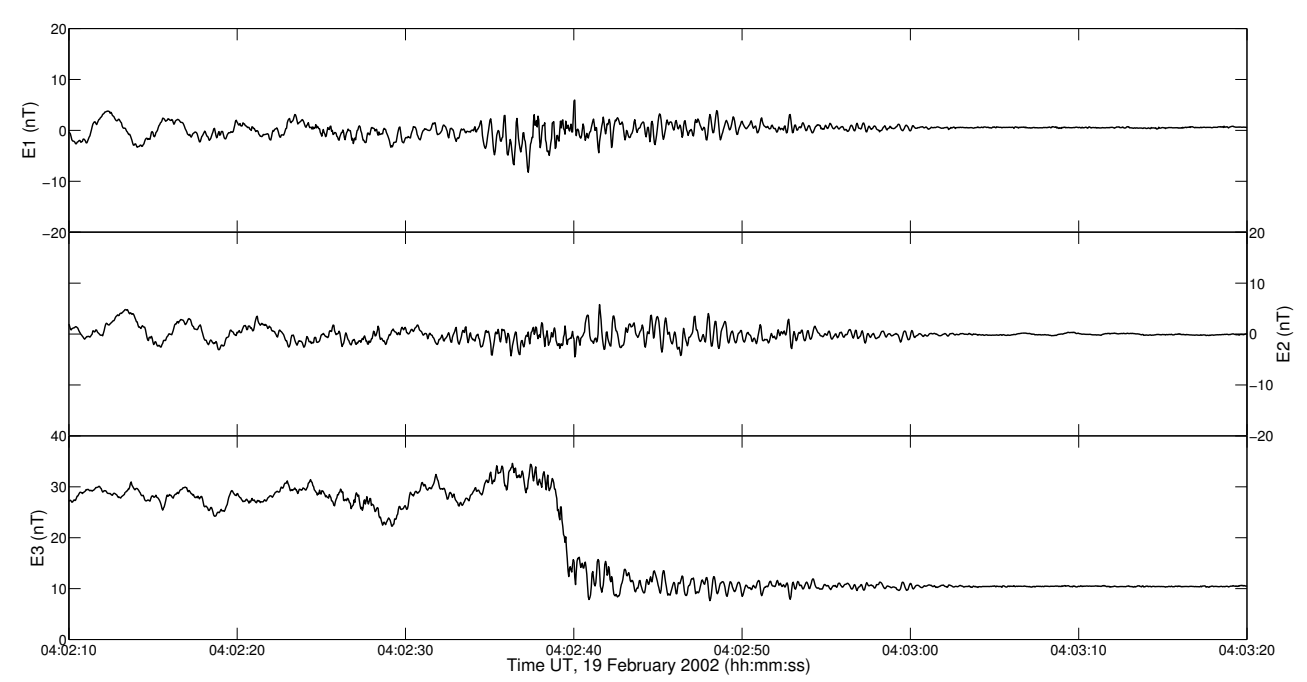

Fig. 1. A shock crossing measured by the Cluster 3 spacecraft on 19 February 2002 at 04:02:40 UTC. The panels represent the measurement in the shock $E_{1,2,3}$ frame where $E_{1}=\hat{\boldsymbol{n}}, E_{2}=\hat{\boldsymbol{n}} \times \boldsymbol{B}_{\mathrm{u}}$ and $E_{3}=\hat{\boldsymbol{n}} \times\left(\hat{\boldsymbol{n}} \times \boldsymbol{B}_{\mathrm{u}}\right)$.

accurately determine the shock velocity. The shock front velocity can have significant impact on the outcome from this study as it is used directly in the transformation to the shock rest frame.

The top panel of Fig. 2 shows the dynamic spectrum of the shock crossing calculated from the FGM data during the time period between 04:02:10 to 04:03:10 UTC. The corresponding $x, y$ and $z$ components of the magnetic field measurements are plotted below in panel $b$. Although obvious from the magnetic field profile in panel $b$, the dynamic spectrum exhibits an evolution from mainly low frequency components before $04: 02: 30$ of $(0.1$ to $0.5 \mathrm{~Hz})$ to higher frequencies $(0.5$ to $4.5 \mathrm{~Hz}$ ) after 04:02:30. The obvious shift from the higher frequency range in the upstream region to the period of significantly lower frequencies (downstream) clearly mark the shock transition region. Interestingly the spectrum shown in panel a of Fig. 2 suggests clear periods of well defined low frequency plasma waves upstream of the shock front between 04:02:45 and 04:03:00 UTC. These waves are identified from the packets of increased frequency components which are significantly elevated above the ambient background level. The well structured perturbations recorded by the magnetic field measurements during this period also correspond very well to these wave packets between 04:02:40 and 04:20:50. What is crucial here is that the small separation vector between $\mathrm{C} 3$ and $\mathrm{C} 4$ parallel to the GSE $x$ direction permits simultaneous observation of the same wave packets upstream of the shock transition. These well defined waves, and the small spacecraft separation between $\mathrm{C} 3$ and $\mathrm{C} 4$ suggest that this shock is an ideal candidate for the application of the method discussed previously and defined by Eq. (1).

Figure 3 shows a more detailed plot of the magnetic field profile of the waves measured along the $x$ component upstream of the shock during the time period
03:45:30-03:45:55 UTC. Panels a and b show the separate $\mathrm{C} 3$ and $\mathrm{C} 4$ measurements whereas panel c underneath represents the same data, however the $\mathrm{C} 4$ (black) measurements have been shifted forward in time by 0.2 seconds to correlate with the $\mathrm{C} 3$ (red) observations. Panel c of Fig. 3 shows that the waves propagate from $\mathrm{C} 4$ to $\mathrm{C} 3$ which interestingly suggests a propagation direction pointed towards the shock front. The separation between these observations is $32 \mathrm{~km}$ which suggests a propagation velocity of $180 \mathrm{~km} \mathrm{~s}^{-1}$ in the spacecraft frame and $148 \mathrm{~km} \mathrm{~s}^{-1}$ in the shock rest frame. The angle between the shock normal and the $x$ axis is only $5.6^{\circ}$, therefore along the shock normal the phase velocity of the waves is directed towards the shock has a magnitude of $165 \mathrm{~km} \mathrm{~s}^{-1}$ in the spacecraft frame, and about $130 \mathrm{~km} \mathrm{~s}^{-1}$ in the shock rest frame.

\section{Wave vector reconstruction and estimation the of experimental dispersion relation}

Minimum variance analysis (Sonnerup and Cahill, 1967) can be used to estimate $\hat{\boldsymbol{k}}$, however this does not provide the magnitude, and also leaves an ambiguity of $\pi$ rad. The problem of ambiguity has already been overcome from the determination of the propagation direction with respect to the position of $\mathrm{C} 3$ and $\mathrm{C} 4$ in the previous section. The application of MVA to data recorded by the $\mathrm{C}_{3}$ and $\mathrm{C}_{4}$ FGM instruments provided directions of $\hat{\boldsymbol{k}}_{\mathrm{C} 3}=[0.75,-0.42,-0.51]$ and $\hat{\boldsymbol{k}}_{\mathrm{C} 4}=[0.77$, $-0.38,-0.51]$, respectively. The agreement between $\hat{\boldsymbol{k}}_{\mathrm{C} 3}$ and $\hat{\boldsymbol{k}}_{\mathrm{C} 4}$ differ only by an angle of only $\approx 2.5^{\circ}$ which suggests a good estimate of $\hat{\boldsymbol{k}}$. Another consideration is the ratio between the intermediate $\left(\lambda_{\text {int }}\right)$ and minimum $\left(\lambda_{\text {min }}\right)$ eigenvalues for $\hat{\boldsymbol{k}}_{\mathrm{C} 3}$ and $\hat{\boldsymbol{k}}_{\mathrm{C} 4}$ which were 18 and 22 , respectively. These ratios also provide confidence in the obtained $\hat{k}$. Based 


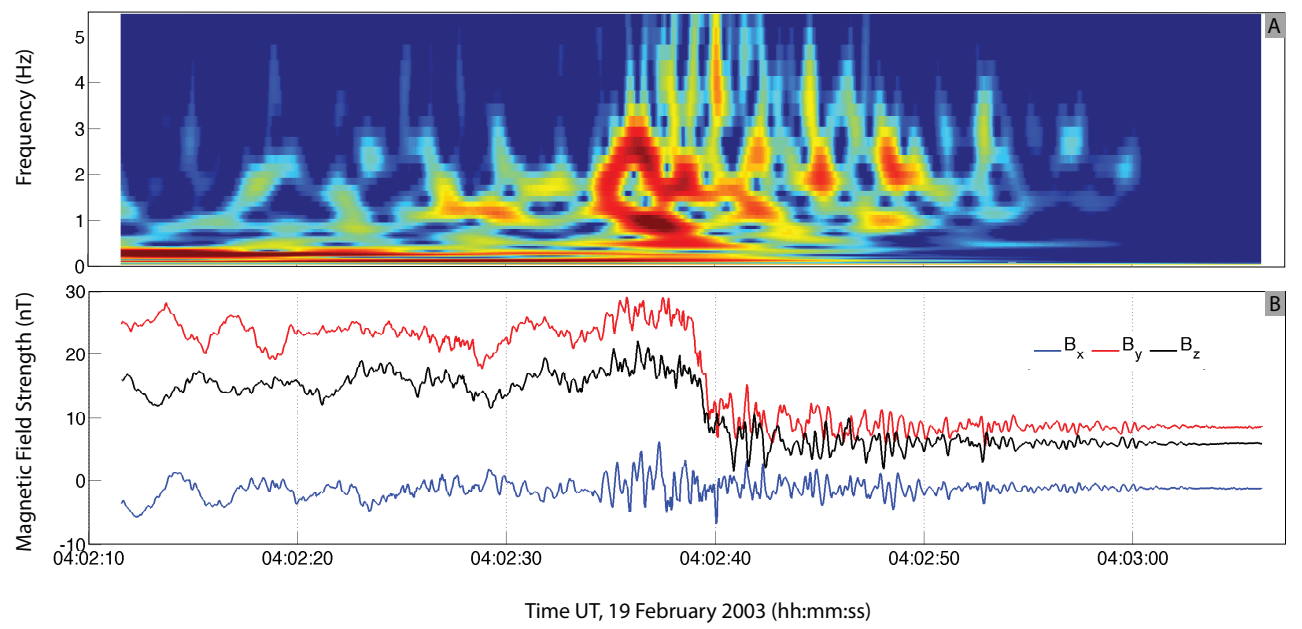

Fig. 2. The frequency spectrum from the wavelet transform of $B_{x}$ measured by the Cluster 3 FGM between 04:02:10 UTC and 04:03:10 UTC. The $x, y$ and $z$ components are plotted underneath over the same time interval.

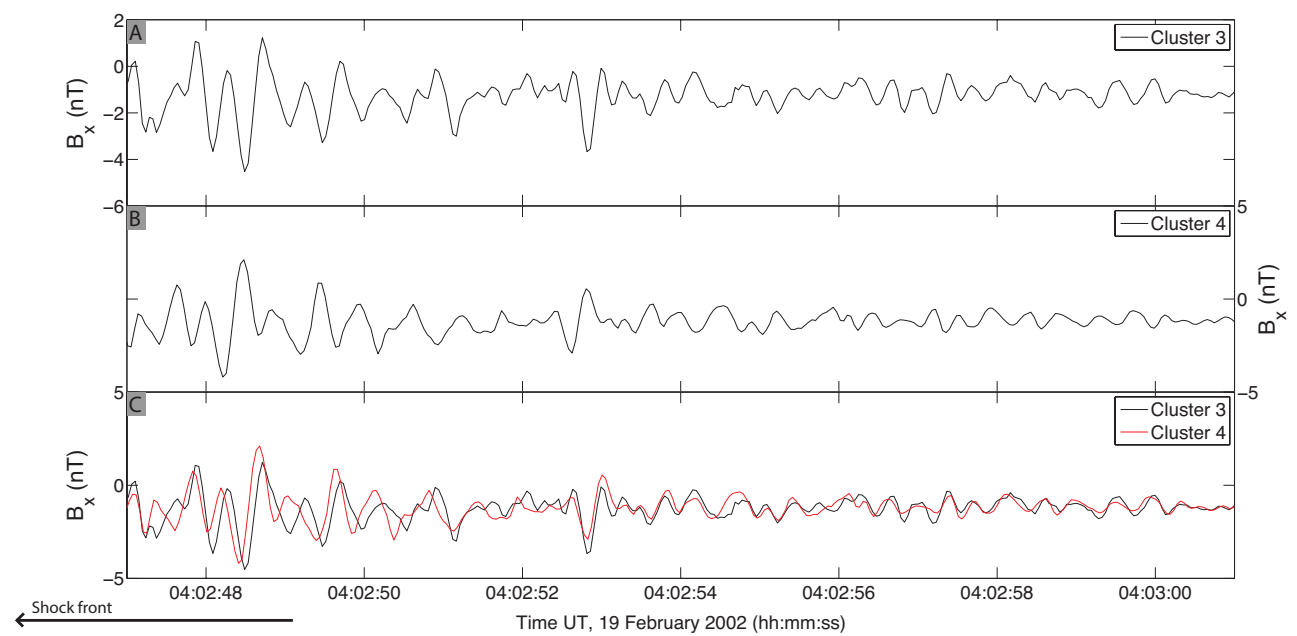

Fig. 3. The FGM $x$ component of low frequency plasma waves measured upstream of the shock front between 03:45:30-03:45:55 UTC. (A) and (B) correspond to measurements made by $\mathrm{C} 3$ and $\mathrm{C} 4$, respectively, whereas (C) below shows $\mathrm{C} 4$ data time shifted forward by $0.2 \mathrm{~s}$ to correlate with the $\mathrm{C} 3$ measurements.

on the mean between $\hat{\boldsymbol{k}}_{\mathrm{C} 3}$ and $\hat{\boldsymbol{k}}_{\mathrm{C} 4}$ the waves are propagating at an oblique angle of $\Theta_{B K} \approx 43.76^{\circ}$ relative to $\boldsymbol{B}_{\mathrm{u}}$. The angle between $\hat{\boldsymbol{k}}$ and $\boldsymbol{R}_{34}$ is $\Theta_{K R} \approx 67.7^{\circ}$ and the wave vector direction differs from the shock normal by $\Theta_{K N} \approx 43.74^{\circ}$. Using the magnetic field in the minimum variance frame, Fig. 4 represents the hodogram calculated over the time interval between 04:02:59.20 and 04:03:00.20 UTC. The waves appear to be circular polarised, whereas the projection of the magnetic field along $\hat{\boldsymbol{k}}$ suggests a correct minimum variance direction.

Figure 5 shows the $f_{1}-k_{\text {sep }}$ spectrum for the waves that are observed upstream of the shock front in Figs. 1, 2 and 3. The spectrum is calculated over the time interval 04:02:52 to 04:03:01 UTC using the $X$ component of the magnetic field shown in Fig. 3. There is a clear relationship between $f_{1}$ and $k_{\text {sep }}$ in the frequency range of $1-4.5 \mathrm{~Hz}$. Naturally, there is an ambiguity of $\pm 2 n \pi$ which in theory extends indefinitely for positive and negative $k_{\mathrm{sep}}$. This is manifested in the $f_{1}-k_{\text {sep }}$ spectrum by repetitive "branches" which are clearly visible in the negative (-) and positive (+) regions of $k_{\text {sep. }}$. The correct branch should correspond with a suitable phase difference that correlates with the time series waveforms. Balikhin et al. (1997) investigated the impact that each branch can have on the frequencies in the PRF. The authors demonstrated that the identification of the proper branch has a notable effect and use of the wrong branch could lead to incorrect identification of the wave mode. The location of the branch is also important, for example, if the branch crosses the $k_{\mathrm{sep}}=0$ line, and $\Theta_{K R}$ is not perpendicular, then this implies an infinite phase velocity. In our case, the phase difference between the $\mathrm{C}_{3}$ and $\mathrm{C}_{4}$ measurements is clearly less than $2 \pi$ which suggests the correct branch should be $(+)$. 

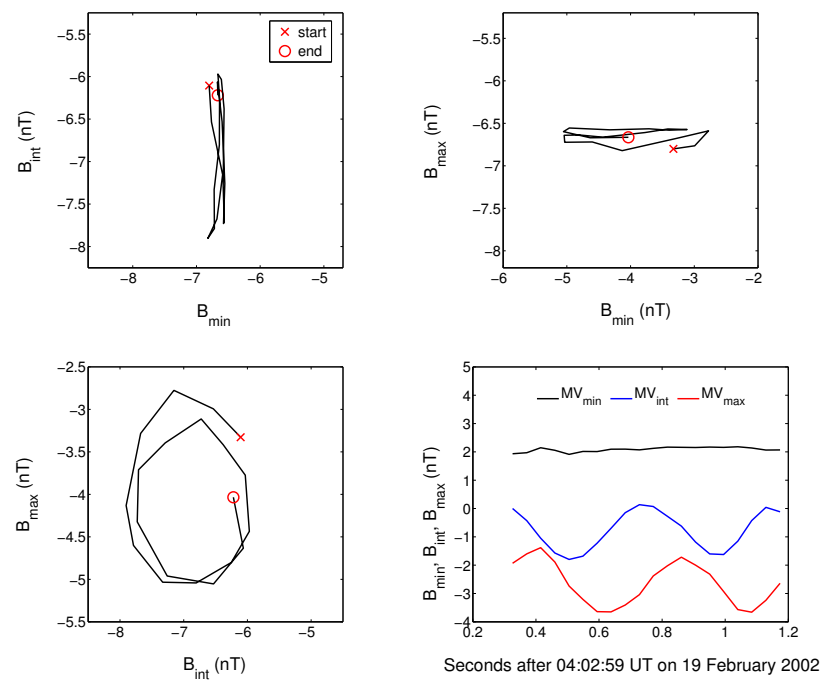

Fig. 4. Hodogram calculated from the $\mathrm{C}_{3}$ FGM data over the time period from 04:02:59.20 to 04:03:00.20 UTC.

The (+) branch also represents waves that propagate from $\mathrm{C}_{4}$ to $\mathrm{C}_{3}$ which correspond to the time series measurements.

The correct value of $k_{\text {sep }}$ is identified by its maximum value, at each observed frequency in the range $1.5 \mathrm{~Hz} \leq f_{1} \leq$ $4.5 \mathrm{~Hz}$. Due to the non-exact thickness of the branch, some error could be introduced by the inaccurate selection of $k_{\text {sep }}$. Although the correct value of $k_{\text {sep }}$ is assumed to correspond to the maximum, an estimate of the error can be provided by introducing variation about the maximum peak. This variation is calculated as the value one mean lower than the maximum. Therefore, at each $f_{1}$, there are three values of $k_{\text {sep. }}$. The first corresponds to the peak $k_{1}$, and the remaining two are the upper and lower limits $\left(k_{\min }\right.$ and $\left.k_{\max }\right)$ either side of the peak, respectively.

There is now sufficient knowledge of the wave properties to calculate the magnitude of the wave vector $|\hat{\boldsymbol{k}}|$ using Eq. (1). Table 1 provides a summary of the complete wave vectors for each corresponding frequency.

The dispersion relation relates the frequencies in the PRF to each corresponding wave vector. Therefore, firstly, this requires the application of the Doppler relation to transform the observed frequencies to those in the PFR.

$\omega_{1}=\omega+\boldsymbol{K}\left(\omega_{1}\right) \cdot \boldsymbol{V}_{\mathrm{u}}$

In the above expression $\omega$ and $\omega_{1}$ represent the frequencies in the spacecraft and PRF, respectively, and $\boldsymbol{V}_{\mathrm{u}}$ is the upstream plasma velocity in the GSE frame. The magnitude of each wave vector which has a corresponding frequency has been estimated using Eq. (1) which are listed in Table 1. The PRF frequencies and each associated wave vector form the experimental dispersion relation for these waves. It is also possible to calculate the theoretical whistler dispersion relation based on $\Theta_{B K}$ which is described by the following expression

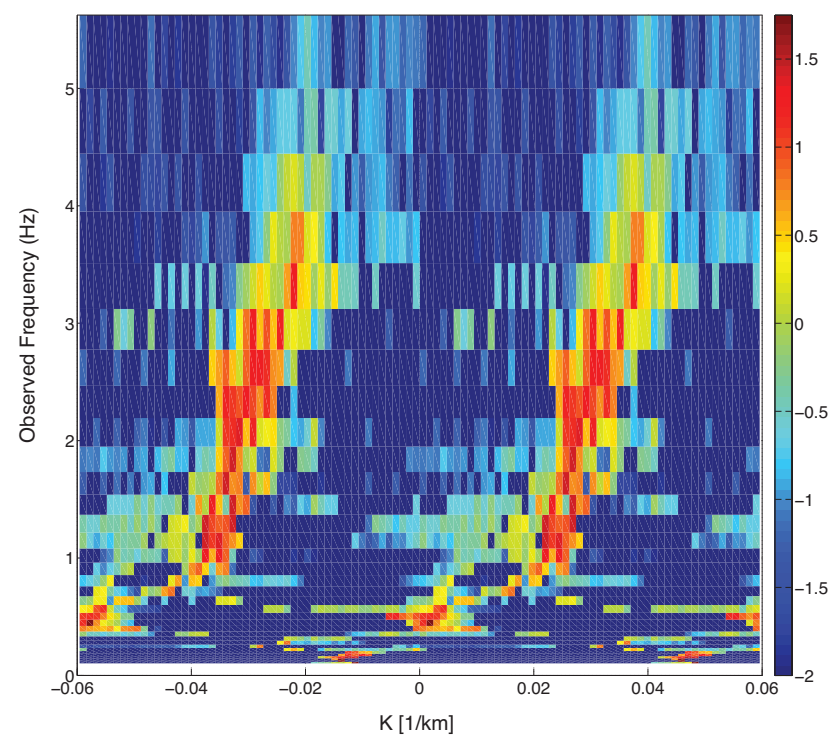

Fig. 5. The $f_{1}-k_{\text {sep }}$ spectrum for waves upstream of the shock over the time period 04:02:52 to 04:03:01 UTC using the $\mathrm{C}_{3}$ and $\mathrm{C}_{4}$ FGM measurements along the $x$ direction.

$\omega_{\mathrm{wh}}=\frac{\Omega_{\mathrm{ce}}|\boldsymbol{K}| c^{2}}{\omega_{\mathrm{pe}}^{2}} \cos \left(\Theta_{B K}\right)$

Figure 6 shows the plasma rest frame frequencies plotted against $|\boldsymbol{K}|$ together with the theoretical dispersion relation for whistler waves propagating at an angle of $47.76^{\circ}$ with respect to the upstream magnetic field. The horizontal error bars represent the possible error that is carried to the full wave vector estimation from a possible variation of $k_{\mathrm{sep}}$, whereas the vertical error bars show the upper and lower limits imposed on the PRF frequencies which would ensue from the evaluation of Eq. (2) from changes in $\boldsymbol{K}\left(\omega_{1}\right)$.

In both the shock and spacecraft frame, we observe that the phase velocity appears to propagate towards the shock front. In the plasma rest frame the phase velocity is directed upstream and away from the shock front. Propagation towards the shock in the shock frame is implicit evidence that waves may be generated as a result of some instability in the foot (e.g. Wu et al., 1984; Wong and Goldstein, 1988). However, it is also possible that they can result from the non-stationarity of the nonlinear wave structure which can be ejected from the ramp into the upstream region. Due to the high amplitude of such a structure they may be able to propagate upstream even if the small amplitude waves with similar spatial scales are not able to do this. With time, this nonlinear structure can decay into a combination of linear waves that posses inferior velocities and are convected back towards the ramp by the solar wind flow. 
Table 1. The full wave vectors at each corresponding observed frequency.

\begin{tabular}{rrr}
\hline$f_{1}(\mathrm{~Hz})$ & $\boldsymbol{K}(1 / \mathrm{km})$ & $|\boldsymbol{K}|(1 / \mathrm{km})$ \\
\hline 1.0814 & {$[0.0423,-0.0237,-0.0287]$} & 0.0563 \\
1.2166 & {$[0.0427,-0.0239,-0.0289]$} & 0.0568 \\
1.3686 & {$[0.0427,-0.0239,-0.0289]$} & 0.0568 \\
1.5397 & {$[0.0518,-0.0290,-0.0351]$} & 0.0689 \\
1.7322 & {$[0.0496,-0.0278,-0.0336]$} & 0.0661 \\
1.9487 & {$[0.0520,-0.0292,-0.0352]$} & 0.0692 \\
2.1923 & {$[0.0539,-0.0303,-0.0366]$} & 0.0718 \\
2.4664 & {$[0.0567,-0.0318,-0.0384]$} & 0.0755 \\
2.7746 & {$[0.0593,-0.0333,-0.0402]$} & 0.0789 \\
3.1215 & {$[0.0591,-0.0331,-0.0400]$} & 0.0787 \\
3.5117 & {$[0.0729,-0.0409,-0.0494]$} & 0.0971 \\
3.9506 & {$[0.0751,-0.0421,-0.0509]$} & 0.1000 \\
4.4444 & {$[0.0727,-0.0408,-0.0493]$} & 0.0968 \\
\hline
\end{tabular}

\section{Discussion and conclusion}

The main difference between wave properties obtained in the present study based on Cluster data and previous one Balikhin et al. (1997) that was based on the AMPTE UKS AMPTE IRM pair of spacecraft is the direction of the phase velocity in the shock frame. In the AMPTE case, the phase velocity was directed upstream and authors of Balikhin et al. (1997) have been using this to argue that the observed waves are generated in the process of the ramp evolution rather than by instability related to the counter-streaming ions in the foot region. Does the down-stream-ward direction of phase velocity in the present case favour the instability scenario? The answer is negative. It is the direction of wave energy motion that is determined by the group velocity $v_{g}=\frac{\mathrm{d} \omega}{\mathrm{d} k}$ is more important than the direction of phase velocity $\frac{\omega}{k}$. The peculiarity of whistler waves is that the quadratic dispersion is a group velocity which is always exactly twice the the phase velocity. In the spacecraft frame the phase velocity of the observed waves is along the shock. If we assume that the wave phase propagation direction corresponds to the minimum variance as determined using Cluster 3 data $\hat{\boldsymbol{k}}_{\mathrm{C} 3}=[0.75,-0.42,-0.51]$ and that solar wind velocity is along GSE $X$ axis, then the velocity of the solar wind along the minimum variance direction is $267 \mathrm{~km} \mathrm{~s}^{-1}$. The phase velocity in the shock frame and along the minimum variance direction is $149.5 \mathrm{~km} \mathrm{~s}^{-1}$.

A proposed source of whistler waves upstream of the shock is proton beam instabilities. However, the model by Wong and Goldstein (1988) for whistler waves propagating at oblique angles to the magnetic field suggests that the plasma rest frame frequencies should be around $20 \Omega_{\text {ci }}$. In this case, the plasma rest frame frequencies range from 21 to over $50 \Omega_{\mathrm{ci}}$. It is therefore unlikely that the whistler waves observed in this case originate from proton beam instabilities.

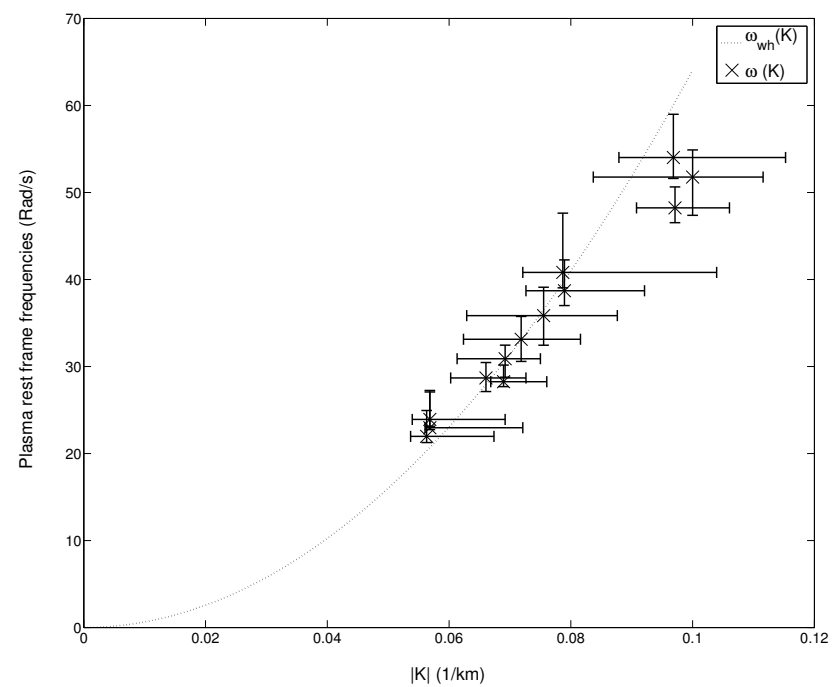

Fig. 6. The dispersion relation for the wave vectors in Table 1 in the $\mathrm{PRF}$ and the theoretical whistler dispersion relation (dotted line) for Whistler waves propagating at an angle of $\Theta_{B K}=43.76^{\circ}$ to the upstream magnetic field.

The temperature anisotropy instability has also been considered as a mechanism for the generation of whittler waves by Wu et al. (1984). However, for sufficient growth rates the range of $\boldsymbol{k}$ vectors would have to be much greater than what was observed in this study. Therefore, this mechanism is unlikely to be responsible.

The kinetic cross field streaming instability has also been attributed to the generation of whistler waves (Wu et al., 1984). For waves propagating at angles of $\Theta_{b k} \approx 30^{\circ} \rightarrow 60^{\circ}$ the frequencies in the plasma frame should be in the range $0.5-1 \omega_{L H}$ at the maximum growth rate. Where $\omega_{L H}=$ $\left[\left(\Omega_{\mathrm{ci}} \omega_{\mathrm{ce}}\right)^{-1}+\omega_{\mathrm{pi}}^{-2}\right]^{-1 / 2}$ is the lower hybrid frequency. In the case of these results the plasma frame frequencies are 0.5$1.2 \omega_{L H}$. The oblique propagation angle, and the frequency ranges suggest that the kinetic cross stream instability could be responsible for the generation of these waves.

Gyro phase bunched ions produced by the shock have been suggested as a local generation mechanism (Gurgiolo et al., 1993) of whistler waves observed upstream of the shock front. Although this process is cannot be discounted, from these observations it is reasonable to postulate that the waves were generated by some process at the shock transition and not locally.

Another generation for whistler waves is attributed to the macro-dynamics of the shock front. It was shown by Tidman and Northrop (1968) that non-stationarity of the shock front can produce whistler waves that can propagate upstream. The process of nonlinear ramp steepening and overturning has also been known to emit nonlinear wave packets upstream of the shock front (Krasnoselskikh, 1985). The experimental determination of whistler waves was performed by Balikhin et al. (1997) using AMTE data. In this example, 
the generation mechanism was due to the shock macro dynamics. In comparison to these results, their frequencies exceeded $\omega_{L H}$ and therefore, kinetic cross field streaming instability was discounted. The solar wind velocity was also higher than normal on this day at $640 \mathrm{~km} \mathrm{~s}^{-1}$ which is also supported the shock non-stationarity. The angle between the shock normal was $27^{\circ}$ (for this case $\Theta_{b k} \approx 43.7^{\circ}$ ) which is also supportive of the macro dynamic models.

\section{Summary}

To summarise: whistler waves have been observed upstream of the quasi-perpendicular and supercritical terrestrial bow shock on 19 February 2002. The phase velocity is directed towards the shock in both the spacecraft and shock frame, but is directed upstream in the plasma rest frame. We have used the observed phase shift measured between $\mathrm{C} 3$ and $\mathrm{C} 4$ to reconstruct the full wave vector and produce the experimental dispersion relation. Comparison with the theoretical dispersion relation confirms that they are indeed propagating in the whistler mode. The properties of these waves suggest that the source mechanism could be related to the shock macro dynamics or the kinetic cross-stream instability.

Acknowledgements. The authors would like to thank the Cluster instrument teams for providing the datasets used in this study. The work was carried out under financial support from EPSRC and STFC.

Topical Editor M. Gedalin thanks two anonymous referees for their help in evaluating this paper.

\section{References}

Balikhin, M. A., Krasnosel'Skikh, V. V., and Woolliscroft, L. J. C.: Reflection of electrons from the front of a strong quasiperpendicular shock and the generation of plasma waves, Adv. Space Res., 9, 203-206, doi:10.1016/0273-1177(89)90115-4, 1989.

Balikhin, M., Krasnosselskikh, V., and Gedalin, M.: The scales in quasiperpendicular shocks, Adv. Space Res., 15, 247-260, doi:10.1016/0273-1177(94)00105-A, 1995.

Balikhin, M. A., de Wit, T. D., Alleyne, H. S. C. K., Woolliscroft, L. J. C., Walker, S. N., Krasnosel'skikh, V., MierJedrzejeowicz, W. A. C., and Baumjohann, W.: Experimental determination of the dispersion of waves observed upstream of a quasi-perpendicular shock, Geophys. Res. Lett., 24, 787-790, doi:10.1029/97GL00671, 1997.

Balikhin, M., Walker, S., Treumann, R., Alleyne, H., Krasnoselskikh, V., Gedalin, M., Andre, M., Dunlop, M., and Fazakerley, A.: Ion sound wave packets at the quasiperpendicular shock front, Geophys. Res. Lett., 32, L24106, doi:10.1029/2005GL024660, 2005.

Balogh, A., Dunlop, M. W., Cowley, S. W. H., Southwood, D. J., Thomlinson, J. G., Glassmeier, K. H., Musmann, G., Lühr, H., Buchert, S., Acuña, M. H., Fairfield, D. H., Slavin, J. A., Riedler, W., Schwingenschuh, K., and Kivelson, M. G.: The Cluster magnetic field investigation, Space Sci. Rev., 79, 65-91, 1997.
Décréau, P. M. E., Fergeau, P., Kranoselskikh, V., Lévêque, M., Martin, P., Randriamboarison, O., Sené, F. X., Trotignon, J. G., Canu, P., and Mögensen, P. B.: WHISPER, a resonance sounder and wave analyser: performances and perspectives for the Cluster mission, Space Sci. Rev., 79, 157-193, 1997.

Dimmock, A. P., Walker, S. N., Zhang, T. L., and Pope, S. A.: Spatial scales of the magnetic ramp at the Venusian bow shock, Ann. Geophys., 29, 2081-2088, doi:10.5194/angeo-29-20812011, 2011.

Escoubet, C. P., Schmidt, R., and Goldstein, M. L.: Cluster Science and Mission Overview, Space Sci. Rev., 79, 11-32, doi:10.1023/A:1004923124586, 1997.

Fairfield, D. H.: Whistler Waves Observed Upstream from Collisionless Shocks, J. Geophys. Res., 79, 1368-1378, doi:10.1029/JA079i010p01368, 1974.

Farris, M. H., Petrinec, S. M., and Russell, C. T.: The thickness of the magnetosheath - Constraints on the polytropic index, Geophys. Res. Lett., 18, 1821-1824, doi:10.1029/91GL02090, 1991.

Galeev, A. A., Klimov, S. I., Nozdrachev, M. N., Sagdeev, R. Z., and Sokolov, A. I.: The dynamics of the magnetoacoustic oscillation spectrum at the front of a earth bow shock and the mechanism for the excitation of these oscillations, Zhurnal Eksperimentalnoi i Teoreticheskoi Fiziki, 90, 1690-1700, 1986.

Guha, J. K., Judge, D. L., and Marburger, J. H.: Ogo 5 magneticfield data near the Earth's bow shock: A correlation with theory, J. Geophys. Res., 77, 604-610, doi:10.1029/JA077i004p00604, 1972.

Gurgiolo, C., Wong, H. K., and Winske, D.: Low and high frequency waves generated by gyrophase bunched ions at oblique shocks, Geophys. Res. Lett., 20, 783-786, doi:10.1029/93GL00854, 1993.

Hellinger, P., Mangeney, A., and Matthews, A.: Whistler waves in 3D hybrid simulations of quasiperpendicular shocks, Geophys. Res. Lett., 23, 621-624, doi:10.1029/96GL00453, 1996.

Hobara, Y., Balikhin, M., Krasnoselskikh, V., Gedalin, M., and Yamagishi, H.: Statistical study of the quasi-perpendicular shock ramp widths, J. Geophys. Res.-Space, 115, 11106, doi:10.1029/2010JA015659, 2010.

Hoppe, M. M., Russell, C. T., Eastman, T. E., and Frank, L. A.: Characteristics of the ULF waves associated with upstream ion beams, J. Geophys. Res., 87, 643-650, doi:10.1029/JA087iA02p00643, 1982.

Krasnoselskikh, V.: Nonlinear motions of a plasma across a magnetic field, Sov. Phys. Jetp, 62, 282-293, 1985.

Krasnoselskikh, V. V., Kruchina, E. N., Volokitin, A. S., and Thejappa, G.: Fast electron generation in quasiperpendicular shocks and type II solar radiobursts, Astron. Astrophys., 149, 323-329, 1985.

Leroy, M. M., Winske, D., Goodrich, C. C., Wu, C. S., and Papadopoulos, K.: The structure of perpendicular bow shocks, J. Geophys. Res., 87, 5081-5094, doi:10.1029/JA087iA07p05081, 1982.

Ofman, L., Balikhin, M., Russell, C. T., and Gedalin, M.: Collisionless relaxation of ion distributions downstream of laminar quasiperpendicular shocks, J. Geophys. Res.-Space, 114, A09106, doi:10.1029/2009JA014365, 2009.

Orlowski, D. S. and Russell, C. T.: ULF waves upstream of the Venus bow shock - Properties of one-hertz waves, J. Geophys. Res., 96, 11271, doi:10.1029/91JA01103, 1991. 
Orlowski, D. S., Russell, C. T., and Lepping, R. P.: Wave phenomena in the upstream region of Saturn, J. Geophys. Res., 97, 19187, doi:10.1029/92JA01461, 1992.

Papadopoulos, K.: Microinstabilities and anomalous transport, Washington DC American Geophysical Union (Geophysical Monograph Series), 34, 59-90, 1985.

Rème, H., Bosqued, J. M., Sauvaud, J. A., Cros, A., Dandouras, J., Aoustin, C., Bouyssou, J., Camus, T., Cuvilo, J., Martz, C., Médale, J. L., Perrier, H., Romefort, D., Rouzaud, J., D’Uston, C., Möbius, E., Crocker, K., Granoff, M., Kistler, L. M., Popecki, M., Hovestadt, D., Klecker, B., Paschmann, G., Scholer, M., Carlson, C. W., Curtis, D. W., Lin, R. P., Mcfadden, J. P., Formisano, V., Amata, E., Bavassano-Cattaneo, M. B., Baldetti, P., Belluci, G., Bruno, R., Chionchio, G., Di Lellis, A., Shelley, E. G., Ghielmetti, A. G., Lennartsson, W., Korth, A., Rosenbauer, H., Lundin, R., Olsen, S., Parks, G. K., Mccarthy, M., and Balsiger, H.: The Cluster ion spectrometry (CIS) experiment, Space Sci. Rev., 79, 303-350, 1997.

Sagdeev, R. Z.: Cooperative Phenomena and Shock Waves in Collisionless Plasmas, Reviews of Plasma Physics, 4, 23-90, 1966.

Sagdeev, R. Z. and Galeev, A. A.: Nonlinear Plasma Theory, W.A. Benjamin, 1969.
Sonnerup, B. U. O. and Cahill Jr., L. J.: Magnetopause Structure and Attitude from Explorer 12 Observations, J. Geophys. Res., 72, 171-183, doi:10.1029/JZ072i001p00171, 1967.

Tanaka, M. and Papadopoulos, K.: Creation of high-energy electron tails by means of the modified two-stream instability, Phys. Fluids, 26, 1697-1699, doi:10.1063/1.864368, 1983.

Tidman, D. A. and Northrop, T. G.: Emission of Plasma Waves by the Earth's Bow Shock, J. Geophys. Res., 73, 1543, doi:10.1029/JA073i005p01543, 1968.

Wong, H. K. and Goldstein, M. L.: Proton beam generation of oblique whistler waves, J. Geophys. Res., 93, 4110-4114, doi:10.1029/JA093iA05p04110, 1988.

Woolliscroft, L. J. C., Alleyne, H. S. C., Dunford, C. M., Sumner, A., Thompson, J. A., Walker, S. N., Yearby, K. H., Buckley, A., Chapman, S., and Gough, M. P.: The Digital Wave-Processing Experiment on Cluster, Space Sci. Rev., 79, 209-231, 1997.

Wu, C. S., Winske, D., Tanaka, M., Papadopoulos, K., Akimoto, K., Goodrich, C. C., Zhou, Y. M., Tsai, S. T., Rodriguez, P., and Lin, C. S.: Microinstabilities associated with a high Mach number, perpendicular bow shock, Space Sci. Rev., 37, 63-109, doi:10.1007/BF00213958, 1984. 